\title{
Spike-timing dependent plasticity in the striatum
}

\author{
Elodie Fino ${ }^{1,2,3 *}$ and Laurent Venance ${ }^{1,2 *}$ \\ Dynamics and Pathophysiology of Neuronal Networks (Institut National de la Santé et de la Recherche Médicale Unité Mixte de Recherche en Santé 667), Center for \\ Interdisciplinary Research in Biology, Collège de France, Paris, France \\ 2 Université Pierre et Marie Curie, Paris, France \\ ${ }^{3}$ Department of Biological Science, Columbia University, New York, NY, USA
}

\section{Edited by:}

Per Jesper Sjöström, University College London, UK

\section{Reviewed by:}

Antonio Pisani, Università di Roma "Tor Vergata," Italy

David M. Lovinger, National Institutes of Health, USA

\section{*Correspondence}

Laurent Venance, Dynamics and Pathophysiology of Neuronal Networks (Institut National de la Santé et de la Recherche Médicale Unité Mixte de Recherche en Santé 667), College de France, 11 Place Marcelin Berthelot, 75005 Paris, France.

e-mail: laurent.venance@college-defrance.fr;

Elodie Fino, Department of Biological Science, Columbia University, 1212 Amsterdam Avenue, 1002 Fairchild building, New York, NY 10027, USA. e-mail:ef2308@columbia.edu
The striatum is the major input nucleus of basal ganglia, an ensemble of interconnected subcortical nuclei associated with fundamental processes of action-selection and procedural learning and memory. The striatum receives afferents from the cerebral cortex and the thalamus. In turn, it relays the integrated information towards the basal ganglia output nuclei through which it operates a selected activation of behavioral effectors. The striatal output neurons, the GABAergic medium-sized spiny neurons (MSNs), are in charge of the detection and integration of behaviorally relevant information. This property confers to the striatum the ability to extract relevant information from the background noise and select cognitive-motor sequences adapted to environmental stimuli. As long-term synaptic efficacy changes are believed to underlie learning and memory, the corticostriatal long-term plasticity provides a fundamental mechanism for the function of the basal ganglia in procedural learning. Here, we reviewed the different forms of spike-timing dependent plasticity (STDP) occurring at corticostriatal synapses. Most of the studies have focused on MSNs and their ability to develop long-term plasticity. Nevertheless, the striatal interneurons (the fast-spiking GABAergic, NO-synthase and cholinergic interneurons) also receive monosynaptic afferents from the cortex and tightly regulated corticostriatal information processing. Therefore, it is important to take into account the variety of striatal neurons to fully understand the ability of striatum to develop long-term plasticity. Corticostriatal STDP with various spike-timing dependence have been observed depending on the neuronal sub-populations and experimental conditions. This complexity highlights the extraordinary potentiality in term of plasticity of the corticostriatal pathway.

Keywords: spike-timing dependent plasticity, corticostriatal, striatum, GABAergic interneurons, cholinergic interneurons, LTP, LTD, basal ganglia

\section{BASAL GANGLIA AND THEIR MAIN INPUT PATHWAY: THE CORTICOSTRIATAL PATHWAY}

Basal ganglia are involved in the learning and memory of cognitive and motor sequences related to environmental stimuli (Graybiel et al., 1994; Packard and Knowlton, 2002; Graybiel, 2005; Yin and Knowlton, 2006). Learning and memory are believed to be underlie by long-term synaptic efficacy changes (Bliss and Collingridge, 1993; Martin and Morris, 2002; Lynch, 2004; Malenka and Bear, 2004). Accordingly, the long-term plasticity at different key pathways within the basal ganglia, provides a basic mechanism for the function of basal ganglia in procedural learning and memory (Yin et al., 2009). As the main input structure of the basal ganglia (Figure 1), the striatum is regarded as a major site of memory formation for sensorimotor and cognitive associations, indicating the importance of the occurrence of different forms of plasticity at corticostriatal synapses (Calabresi et al., 1996; Mahon et al., 2004; Costa, 2007; Kreitzer and Malenka, 2008; Di Filippo et al., 2009). In addition, corticostriatal plasticity is severely altered in several pathologies affecting basal ganglia (Kreitzer and Malenka, 2008; Calabresi et al., 2009). Therefore, it is essential to understand in which conditions of cortical and striatal activity, long-term plasticity occurs at corticostriatal synapses. It is now well established that the temporal relationship of activity in pre- and post-synaptic neurons is determinant for the induction of activity-dependent long-term plasticity, a process named spike-timing dependent plasticity (STDP) described from insects to mammalian brain structures (Sjöström and Nelson, 2002; Bi and Rubin, 2005; Dan and Poo, 2006; Caporale and Dan, 2008). In this review, we will focus on STDP experiments recently reported at the corticostriatal synapses as a Hebbian synaptic learning rule.

Basal ganglia are composed of six nuclei: two input nuclei, the striatum and the subthalamic nucleus (STN), two output nuclei, the substantia nigra pars reticulata $(\mathrm{SNr})$ and the internal segment of the globus pallidus (GPi), one relay nucleus, the external segment of the globus pallidus (GPe) and one dopaminergic neuromodulatory nucleus, the substantia nigra pars compacta (SNc) (Figure 1). The striatum is the main input nucleus of basal ganglia and receives massive convergent glutamatergic inputs from the cerebral cortex and the thalamus. In turn, the striatum relays the integrated cortical information towards the two basal ganglia output nuclei ( $\mathrm{SNr}$ and GPi), through two anatomo-functional pathways: the direct pathway (cortico-striato-nigral) and the indirect pathway (cortico-striato-pallido-nigral) (Figure 1). In the motor control, the direct and indirect pathways exert opposite influence, respectively, inhibitory and excitatory on basal output nuclei. Therefore, the activation of the direct pathway would initiate or facilitate the movement while the activation of the indirect pathway would constitute a brake. The neuromodulation of different basal ganglia 


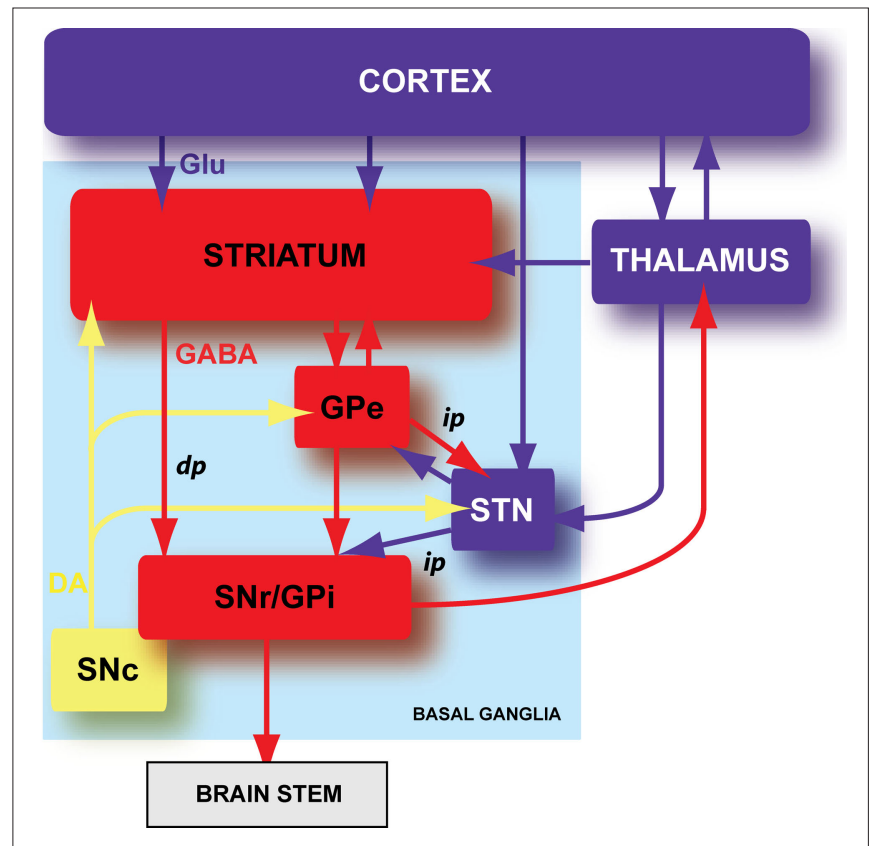

FIGURE 1 | Schematic organization of the basal ganglia. Basal ganglia are an ensemble of tightly interconnected sub-cortical nuclei. In blue are represented the glutamatergic (Glu) structures, in red the GABAergic (GABA) nuclei and in yellow the dopaminergic (DA) nucleus. GPe: external part of the globus pallidus; GPi: internal part of the globus pallidus; SNr: substantia nigra pars reticulata; SNc: substantia nigra pars compacta; dp: direct pathway; ip: indirect pathway.

nuclei by SNc dopaminergic neurons plays a central role since the dopamine brings a motivational side of the cortical information integration in the basal ganglia (Redgrave and Gurney, 2006; Costa, 2007; Schultz, 2007).

Most models of basal ganglia emphasize the importance of the corticostriatal connection. However, the glutamatergic neurons from intralaminar thalamus also innervate the striatum (Groenewegen and Berendse, 1994; Smith et al., 2004). Corticostriatal and thalamostriatal synapses on MSNs appear to be nearly equal in number (Smith et al., 2004), but display different functional characteristics (Smeal et al., 2007; Ding et al., 2008). Because of the lack of experimental data targeting the thalamostriatal long-term plasticity, we will focus on the corticostriatal pathway in this review.

\section{A MARKED ANATOMO-FUNCTIONAL HETEROGENEITY UNDER THE STRIATAL SURFACE}

The striatum displays numerous heterogeneities based, not only on its anatomo-functional organization (striosome versus matrix compartments, and somatosensory/motor/prefrontal projection areas), but also on a cellular diversity (Graybiel, 1990; Groenewegen et al., 1990; Deniau and Thierry, 1997). The striatum is composed for a vast majority ( $95 \%$ in rodents and $80 \%$ in primates) of striatal output neurons, the medium-sized spiny neurons (MSNs). Among MSNs, different populations can be distinguished based on their specific expression of receptors, channels, peptides or modes of communication (Graybiel, 1990; Gerfen, 1992; Nicola et al., 2000; Venance et al., 2004; Vandecasteele et al., 2007). In addition, the striatum also comprises GABAergic and cholinergic interneurons, which tightly regulate MSN excitability and consequently, the corticostriatal information processing. Therefore, the striatum is a highly complex structure and the link between such complexity and the different modes of synaptic plasticity needs to be characterized.

THE STRIATAL OUTPUT NEURONS: THE MEDIUM-SIZED SPINY NEURONS Medium-sized spiny neurons are in charge of the detection and integration of behaviorally relevant information. MSNs, in vivo as well as in vitro, are characterized by their low level of spontaneous activity that can be explained by non-linear electrical membrane properties due to a set of voltage-gated potassium and sodium currents (Nisenbaum et al., 1994; Nisenbaum and Wilson, 1995). These non-linear membrane properties allow an efficient filtering of the small and uncorrelated synaptic events. Consequently, MSNs, quiescent at rest, need strong and correlated cortical inputs to discharge (Calabresi et al., 1987; Nisenbaum and Wilson, 1995). Therefore, MSNs act as coincidence detectors of cortical activity and have the ability to extract relevant information from the background noise. Among MSNs, different sub-populations can be distinguished based on receptors or peptides specific expression. Specifically, MSNs expressing mainly either dopaminergic type-1 (D1) or type-2 (D2) receptors project through, respectively, the direct or the indirect pathway (Figure 1). Such heterogeneity is the most studied at the moment thank to the use of D1-EGFP and D2-EGFP mice that constitute a useful tool to distinguish between MSNs belonging to the direct or indirect pathways (Surmeier et al., 2007; Valjent et al., 2009). However, if MSNs appear to be segregated in mice (D2-GFP staining being restricted to the indirect pathway; Matamales et al., 2009), it exists in rats and primates a significant population (respectively, 30 and $80 \%$ ) of MSNs that project to both direct and indirect pathways (Kawaguchi et al., 1990; Wu et al., 2000; Levesque and Parent, 2005).

\section{THE GABAergic INTERNEURONS}

Three classes of striatal GABAergic interneurons can be distinguished (1) the parvalbumin positive cells (fast-spiking interneurons) (Kawaguchi, 1993), (2) the calretinin positive cells (FigueredoCardenas et al., 1996) (their electrophysiological features remain to be determined) (Tepper and Bolam, 2004) and (3) the neuronal nitric oxide synthase (nNOS) interneurons (persistent and low-threshold spiking cells, PLTS) (Kawaguchi, 1993). Fast-spiking GABAergic interneurons exert a powerful inhibitory weight (Figure 2A) since they can delay or prevent the emission of an action potential in MSNs (Kita, 1996; Plenz and Kitai, 1998; Koos and Tepper, 1999). These interneurons preferentially contact MSNs on the soma (Kita et al., 1990; Bennett and Bolam, 1994), which reinforces the inhibitory shunt. Because they also receive cortical inputs (Bennett and Bolam, 1994; Ramanathan et al., 2002; Mallet et al., 2005), fast-spiking interneurons could provide a feed-forward mechanism increasing the selectivity of MSN responsiveness to cortical inputs and the funneling of the corticostriatal information processing. Compared to fast-spiking interneurons, nNOS interneurons contact MSN mainly on the neck of the spines and display a lower number of synapses (Kubota and Kawaguchi, 2000). Anatomical and functional existence of synapses between cortical glutamatergic afferents and striatal nNOS interneurons has been reported (Vuillet et al., 1989; Fino et al., 


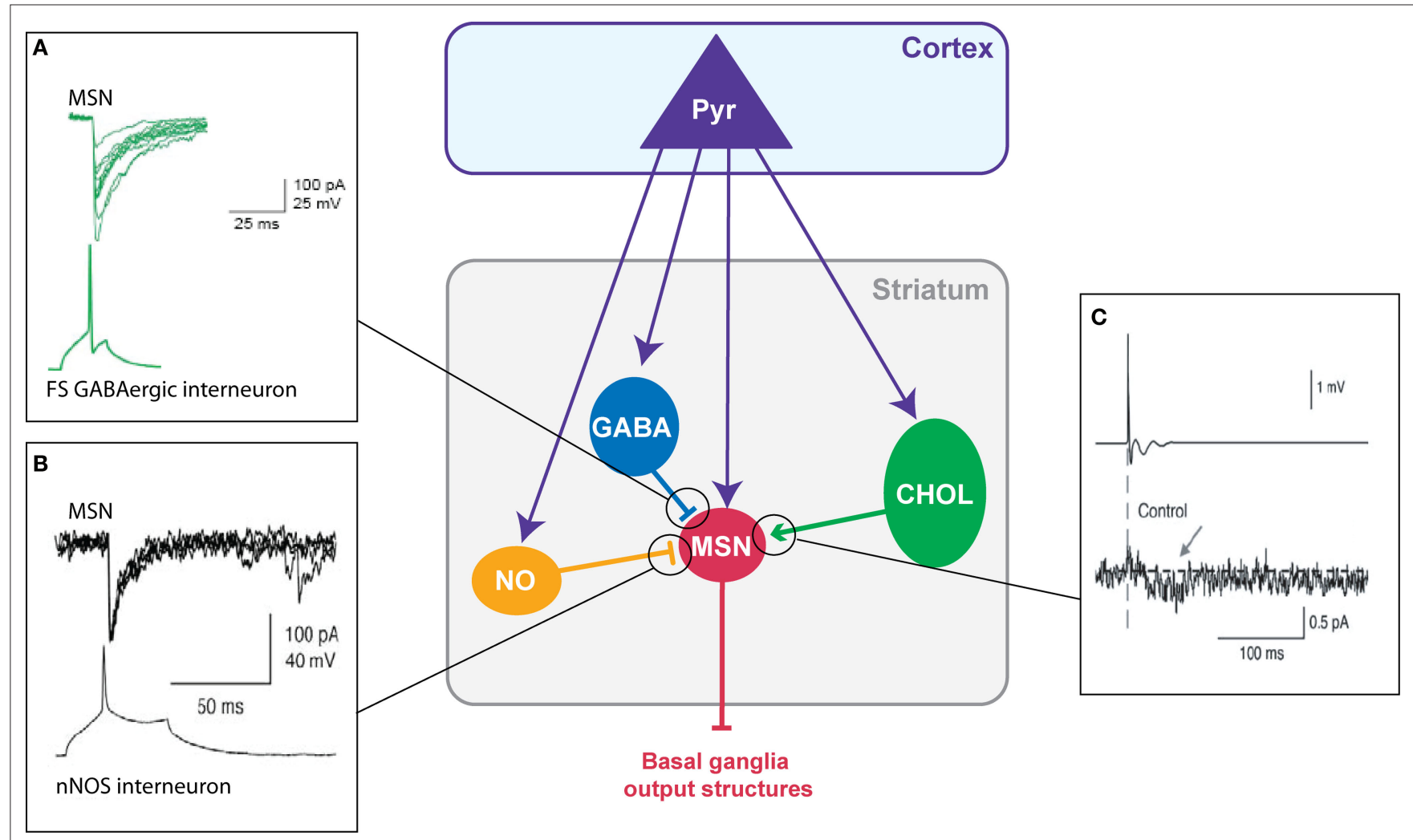

FIGURE 2 | Chemical transmission between striatal neurons. (A) Fastspiking GABAergic interneurons have a strong inhibitory weight on MSNs: an action potential in the interneuron evokes an IPSC in the MSN (Adapted from Tepper et al., 2004). (B) Dual patch-clamp recording illustrating the inhibitory action of nNOS interneurons on MSNs (Adapted from Tepper and Bolam, 2004) (C) Illustration of the excitatory effect of cholinergic interneurons on MSNs: an extracellularly evoked action potential in a cholinergic interneuron evokes an EPSC in the MSNs (Adapted from Lin et al., 2004). 2009b). In addition to NOS, nNOS interneurons express the synthetic enzyme for GABA (Vuillet et al., 1990; Kawaguchi, 1993; FigueredoCardenas et al., 1996; Kubota and Kawaguchi, 2000) and have been characterized functionally as GABAergic cells that efficiently inhibit MSNs (Figure 2B) (Koos and Tepper, 1999; Tepper and Bolam, 2004). They also exert an inhibitory influence on MSNs via NO release (Sardo et al., 2002; West and Grace, 2004). NO modulates the MSN synaptic plasticity since the blockade of NO synthesis or the application of $\mathrm{NO}$ precludes or promotes, respectively, the induction of a long-term depression (LTD) after a high-frequency stimulation (HFS) (Calabresi et al., 1999; Sergeeva et al., 2007).

\section{THE CHOLINERGIC INTERNEURONS}

Cholinergic interneurons receive cortical inputs (Thomas et al., 2000; Reynolds and Wickens, 2004; Fino et al., 2008) and regulate the excitability of MSNs. Cholinergic cells fire tonically in vivo and provide a synchronized signal throughout the striatal network in response to sensory cues predictive of reward (Aosaki et al., 1994; Kimura et al., 2003; Morris et al., 2004; Apicella, 2007). Indeed, dopamine controls the discharge activity of cholinergic cells. Cholinergic interneurons modulate MSNs activity through various muscarinic receptors (Bennett and Bolam, 1994). MSNs of the direct and indirect pathways bear distinct patterns of muscarinic receptor expression. MSNs of the direct pathway express both muscarinic type-1, M1, (excitatory) and type-4, M4, (inhibitory) receptors and MSNs of the indirect pathway express M1 receptors (Acquas and DiChiara, 2002). Therefore, a same change in the cortical synaptic weight of cholinergic interneurons should result in opposite effects in the two sub-populations of MSNs. Nevertheless, muscarinic agonists (acetylcholine or muscarine) have mainly an excitatory effect on the MSNs by increasing their activity (Perez-Rosello et al., 2005) or the EPSC amplitude (Lin et al., 2004; Pakhotin and Bracci, 2007) due to M1 receptor activation (Figure 2C). In addition, acetylcholine acts on nicotinic receptors mainly located within the striatum on dopaminergic terminals (Clarke and Pert, 1985; Exley and Cragg, 2008). Finally, cholinergic interneurons also strongly influence the corticostriatal HFS-induced plasticity on MSNs since they favor the induction of a long-term potentiation (LTP) (Centonze et al., 2003; Surmeier et al., 2007) via the activation of muscarinic receptors whereas nicotinic receptor activation contributes to the induction of LTD (Partridge et al., 2002).

Therefore, besides MSNs, it is primordial to take into account the synaptic plasticity at striatal interneurons since they are directly connected to the cerebral cortex and control striatal microcircuits as well as corticostriatal information processing.

\section{SPIKE-TIMING DEPENDENT PLASTICITY IN STRIATAL OUTPUT NEURONS}

Corticostriatal plasticity has been extensively studied with classical conditioning protocols using low $(1 \mathrm{~Hz})$, medium $(10 \mathrm{~Hz})$ or high-frequency stimulation $(100 \mathrm{~Hz}$ ) (Mahon et al., 2004; Kreitzer 
and Malenka, 2008; Di Filippo et al., 2009). HFS of striatal afferents applied within the striatum or the corpus callosum leads to LTD at MSN synapses (Calabresi et al., 1992a), but to LTP when the electrical stimulation was performed within the cortex (Fino et al., 2005). HFS-induced LTD relies on group-1 metabotropic glutamate receptors, dopamine D2 receptors, voltage-sensitive calcium channels and CB1 receptor activation (Di Filippo et al., 2009; Kreitzer and Malenka, 2008). Corticostriatal LTP was induced by cortical HFS (Fino et al., 2005), while the removal of extracellular magnesium was requested to observe a LTP when stimulation were performed into the striatum or the corpus callosum (Calabresi et al., 1992a,b). HFS-induced LTP requires NMDA receptor activation (Di Filippo et al., 2009; Kreitzer and Malenka, 2008).

STDP is based on the quasi-coincidence between pre- and postsynaptic activity within several milliseconds time scale. Despite numerous studies addressing the corticostriatal plasticity, to this day STDP at the corticostriatal synapses onto MSNs has been explored in only three different studies (Fino et al., 2005; Pawlak and Kerr, 2008; Shen et al., 2008). STDP was reported to be a very efficient phenomenon occurring at corticostriatal synapses since it occurred in roughly $80 \%$ of the cells (Fino et al., 2005; Pawlak and Kerr, 2008). Depending on the experimental conditions, different spike-timing dependences have been reported. Using a classical STDP protocol (100 paired stimulations at $1 \mathrm{~Hz}$ ) without pharmacological manipulation, a "reversed" time-dependence is observed (Fino et al., 2005), compared to those described so far in other mammalian brain structures (Markram et al., 1997; Dan and Poo, 2004, 2006). Indeed, post-pre pairings induced STDP-LTP ( $\mathrm{t}$-LTP) and pre-post pairings induced STDP-LTD ( $\mathrm{t}$-LTD) in MSNs (Fino et al., 2005) (Figure 3A). Conversely, paired stimulations at $0.1 \mathrm{~Hz}$ with blockade of $\mathrm{GABA}_{\mathrm{A}}$ transmission, was found to evoke a t-LTD after post-pre pairings and a t-LTP following pre-post pairings (Pawlak and Kerr, 2008) (Figure 3B). Recently, another study using theta-burst protocol associated to a blockade of GABA transmission reported a lack of STDP at D1 receptor expressing MSNs after post-pre pairings (Shen et al., 2008). Therefore, the striatal anatomo-functional heterogeneity could be revealed with specific experimental conditions, highlighting the fantastic complexity of the striatum. Depending on the experimental conditions, different receptors and intracellular pathways appear to underlie the MSN STDP. Indeed, t-LTP and t-LTD induced by a $0.1 \mathrm{~Hz}$ pairings relied on one coincidence detector, the NMDA receptor, associated with D1 receptor activation (Pawlak and Kerr, 2008). A different picture is obtained with the t-LTP and t-LTD induced by $1 \mathrm{~Hz}$ pairings, since they are mediated by independent signaling mechanisms, each one controlled by distinct coincidence detectors. Namely, t-LTP relies on the NMDA receptor, while t-LTD requires distinct coincident detectors: the phospholipase C $\beta$ (PLC $\beta$ ), the inositol-triphosphate receptor ( $\left.\mathrm{IP}_{3} \mathrm{R}\right)$-gated calcium stores and the diacylglycerol lipase $\alpha$ (DGL $\alpha$ ) (Fino et al., 2010). PLC $\beta$ activation is controlled by group-I metabotropic glutamate receptors, type-1 muscarinic receptors and voltage-sensitive calcium channels activities. The activation of PLC $\beta, \mathrm{IP}_{3}$ Rs and DGL $\alpha$ leads to robust retrograde endocannabinoid signaling mediated by 2 -arachidonoyl-glycerol and cannabinoid CB1 receptors (Fino et al., 2010). Similarly, in the theta-burst based STDP, t-LTP is NMDA receptor and $\mathrm{D} 1$ receptor activation dependent whereas t-LTD is dependent

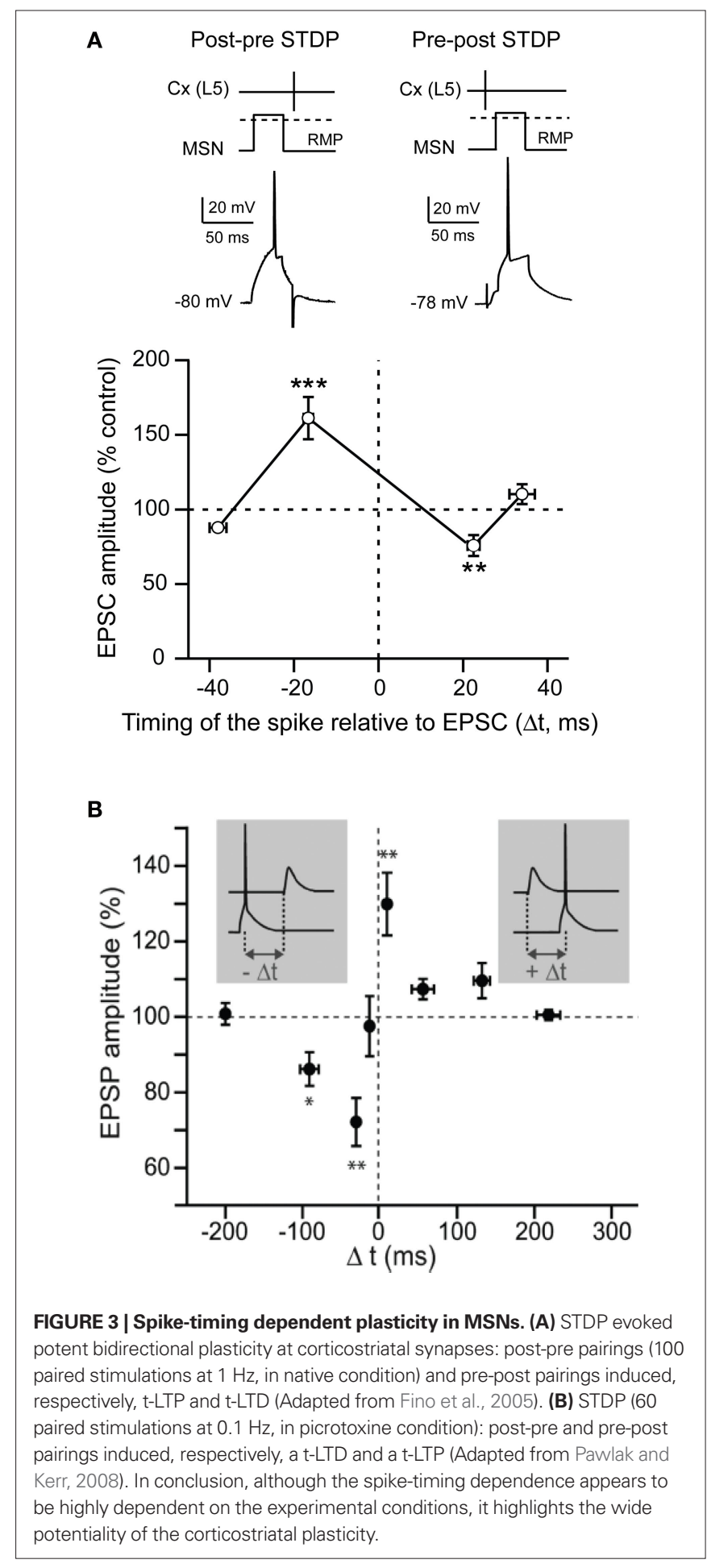

on mGluRs, endocannabinoids and dopaminergic $\mathrm{D} 2$ receptor activation (Shen et al., 2008). The signaling pathways involved in corticostriatal STDP appear to be similar to those requires for HFS- or LFS-induced corticostriatal plasticity. These observations suggest that the corticostriatal pathway is a highly responsive system, in which different signaling cascades are involved, depending on the corticostriatal paired activity. 
The differences between these studies, instead of being presenting as conflicting, should be seen as many promising leads to decipher the extraordinary complexity of the striatum and its potentiality to display various corticostriatal plasticity. The first important observation is that different experimental conditions and different STDP protocols can lead to long-term plasticity. In any cases, discrepancies between the results could be explained by various experimental conditions. First, the different species used (mice versus rats) may matter, especially when analyzing the direct and indirect pathways. Second, the location of the electrical stimulation of the "presynaptic" element is performed either in the layer 5 of the somatosensory cortex (Fino et al., 2005), or in the corpus callosum (Pawlak and Kerr, 2008), or directly within the striatum at the MSN dendrites (Shen et al., 2008). In addition, the STDP protocols are different since they consist in the emission of a single action potential in the post-synaptic MSNs (Fino et al., 2005; Pawlak and Kerr, 2008) or a burst of action potentials (Shen et al., 2008). The frequency of the pairing varies between $0.1 \mathrm{~Hz}$ (Pawlak and Kerr, 2008), $1 \mathrm{~Hz}$ (Fino et al., 2005) and $5 \mathrm{~Hz}$ (Shen et al., 2008). Finally, and most importantly, the "classical" time-dependence of corticostriatal STDP has been observed while the GABAergic transmission was blocked (Pawlak and Kerr, 2008; Shen et al., 2008) and the "reversed" STDP without any blockade of GABAergic circuits (Fino et al., 2005). The GABAergic microcircuits, including GABAergic interneurons and MSN collaterals, play a crucial role in the local interactions in the striatum (Koos and Tepper, 1999; Tepper et al., 2004; Venance et al., 2004). Therefore, it remains necessary to evaluate the contribution of GABAergic circuits in the corticostriatal spike-timing dependence.

\section{STDP IN STRIATAL INTERNEURONS}

Attention has been merely focused on corticostriatal long-term plasticity in MSNs, the striatal output neurons. Nevertheless, as previously mentioned, striatal interneurons regulate MSN excitability and are contacted monosynaptically by glutamatergic afferents from the cortex; consequently, they are expected to play a determinant role in the corticostriatal information processing. Despite their role, the induction of long-term plasticity onto striatal interneurons has been barely addressed. It has been reported that HFS in the corpus callosum induced a LTP in cholinergic interneurons (Suzuki et al., 2001; Bonsi et al., 2004). More recently, it as been shown that the three types of striatal interneurons, cholinergic, fast-spiking GABAergic and nNOS interneurons, were able to develop long-term plasticity following STDP pairings (100 paired stimulations at $1 \mathrm{~Hz}$, in native conditions) (Figure 4) (Fino et al., 2008, 2009b). Fast-spiking GABAergic interneurons display a STDP with a spike-timing dependence similar to those described in the cerebral cortex or in the hippocampus. Indeed, post-pre pairings induced t-LTD and pre-post pairings induced t-LTP in GABAergic interneurons (Figure 4A).t-LTP as well as t-LTD were dependent of the activation of NMDA receptors (Fino et al., 2008). Concerning cholinergic interneurons, we observed a partially reversed STDP: post-pre pairings induced t-LTP as well as t-LTD (with a majority of t-LTP) whereas pre-post pairings induced exclusively t-LTD (Figure4B). Interestingly, the state of excitability of cholinergic interneurons is correlated to the induction of either a t-LTD or a t-LTP after post-pre pairings (Fino et al., 2008). Pharmacological

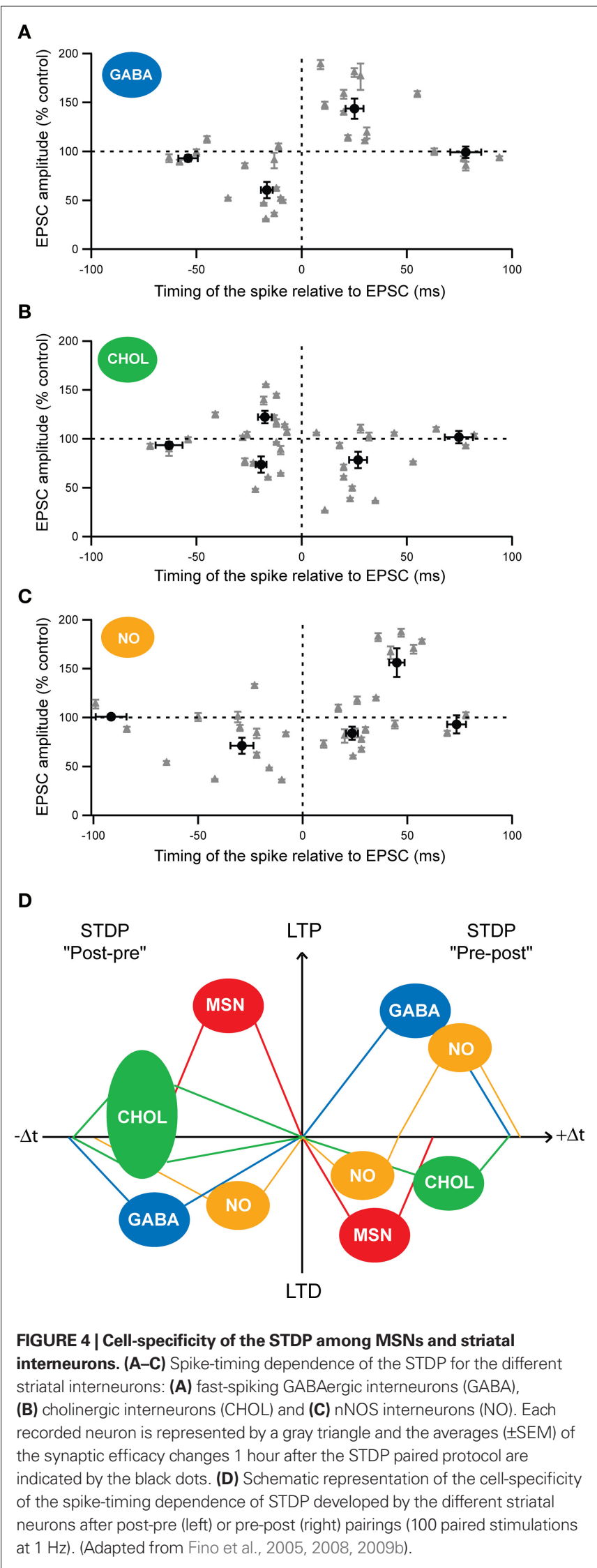

B

FIGURE 4 | Cell-specificity of the STDP among MSNs and striatal interneurons. (A-C) Spike-timing dependence of the STDP for the different striatal interneurons: (A) fast-spiking GABAergic interneurons (GABA), (B) cholinergic interneurons (CHOL) and (C) nNOS interneurons (NO). Each recorded neuron is represented by a gray triangle and the averages ( \pm SEM) of the synaptic efficacy changes 1 hour after the STDP paired protocol are of the spike-timing dependence of STDP developed by the different striatal at $1 \mathrm{~Hz}$ ). (Adapted from Fino et al., 2005, 2008, 2009b). 
experiments have indicated that t-LTP was NMDA receptor activation dependent whereas t-LTD was dependent of the activation of group-1 glutamate metabotropic receptors (mGluR) (Fino et al., 2008). Concerning the nNOS interneurons, STDP was atypical since it displayed an asymmetric time-dependence: t-LTD was induced by post-pre $(-65<\Delta \mathrm{t}<0 \mathrm{~ms})$ but also by "early" pre-post sequences $(0<\Delta \mathrm{t}<+30 \mathrm{~ms})$, whereas $\mathrm{t}-\mathrm{LTP}$ was exclusively induced by "late" pre-post sequences $(+30<\Delta \mathrm{t}<+65 \mathrm{~ms}$ ) (Figure 4C) (Fino et al., $2009 \mathrm{~b})$. This constitutes the first example of a STDP with such a form of asymmetric plasticity (t-LTD) spanning over negative and positive $\Delta t$ and followed by the other form of synaptic efficacy changes (t-LTP). Concerning the nNOS interneurons, the signaling pathways underlying the STDP remain to be characterized.

These results reveal the existence of a marked cell-specificity of the spike-timing dependence (Figure 4D) and of the signaling cascades among striatal neuronal populations. Such cell-specificity of STDP has also been observed in the cochlear nucleus where the principal cells and the glycinergic interneurons display different STDP time-dependence (Tzounopoulos et al., 2004) and in the cortex, with different time-dependence of pyramidal cells and layer 4 spiny stellate cells (Markram et al., 1997; Egger et al., 1999).

\section{CONSEOUENCES OF THE STRIATAL STDP CELL-SPECIFICITY}

The common feature within all the different striatal neuronal subtypes is that STDP can be induced at a very high occurrence in MSNs (90\%), fast-spiking GABAergic (95\%), cholinergic (86\%) and nNOS interneurons (90\%) (Fino et al., 2005, 2008, 2009b; Pawlak and Kerr, 2008).

Among striatal neurons, the temporal window in which STDP is induced, displays a marked cell-specificity. Indeed, it is narrower for MSNs $(-30<\Delta \mathrm{t}<+30 \mathrm{~ms})$ than for fast-spiking GABAergic
$(-40<\Delta \mathrm{t}<+60 \mathrm{~ms})$, cholinergic $(-50<\Delta \mathrm{t}<+60 \mathrm{~ms})$ and nNOS $(-65<\Delta \mathrm{t}<+65 \mathrm{~ms}$ ) interneurons (Fino et al., 2005, 2008, 2009b). The temporal window for STDP induction is larger for the different striatal interneurons than MSNs. Functionally, this means that, for $\Delta \mathrm{t}<-30 \mathrm{~ms}$ and for $\Delta \mathrm{t}>+30 \mathrm{~ms}$, the synapses onto interneurons are still subject to long-term plasticity whereas the synaptic efficacy changes at the MSNs themselves become unaffected. In addition, interneurons are recruited by cortical afferents slightly before the MSNs (Mallet et al., 2005; Fino et al., 2008), meaning that they are able to influence directly the cortical information integration by the MSNs. These different aspects highlight the impact of the interneurons on the control of the striatal output.

Considering the cell-specificity of the STDP together with the local interactions between striatal interneurons and MSNs, we propose a simplified scheme of the impact of the interplay of the different STDP on the striatal output (Figure 5). It should be noted that this scheme is based on data obtained in the same experimental conditions: the horizontal brain slices without affecting the GABAergic transmission with identical STDP protocol (100 paired stimulations at $1 \mathrm{~Hz}$ ). A post-pre pairing induces a t-LTP in MSN, a t-LTD in GABAergic and nNOS interneurons and both forms of plasticity in cholinergic interneurons (with a majority of t-LTP) (Figures 4D and 5A). Conversely, after a pre-post pairing, MSNs and cholinergic interneurons develop a t-LTD while GABAergic and nNOS interneurons display a strictly opposite STDP orientation, a t-LTP (Figures 4D and 5B). The question is: how this cellspecificity of STDP acts to influence the striatal output? To answer this question, we need to consider the local interactions between the striatal interneurons and MSNs. As previously described in this review, local interactions between interneurons and MSNs are

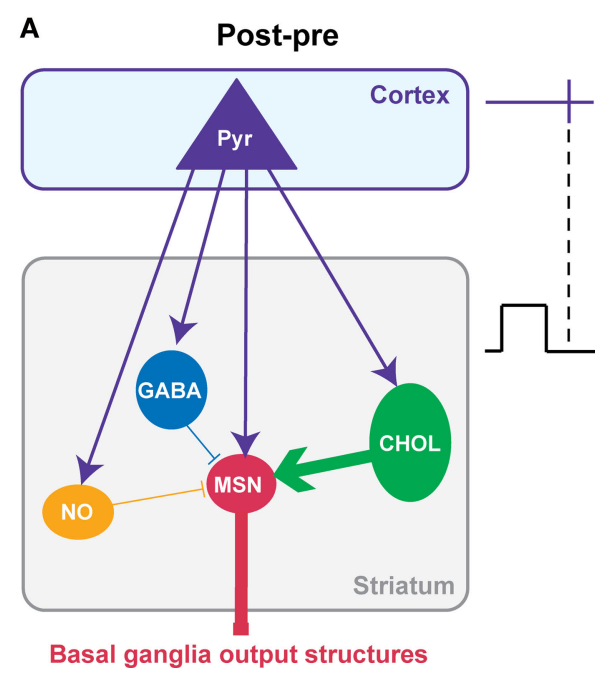

FIGURE 5 | Putative functional consequences of corticostriatal STDP cell-specificity on the striatal output. A simplified model of the interplay of the STDP occurring at the different striatal neuron populations taking into account the main synaptic interactions between striatal interneurons and MSNs. The effects of the combined STDP on the striatal output were considered after post-pre (A) or pre-post pairings (B). (A) Post-pre pairings induced a depression of the GABAergic inhibition from interneurons while reinforcing the synaptic efficacy of MSNs and

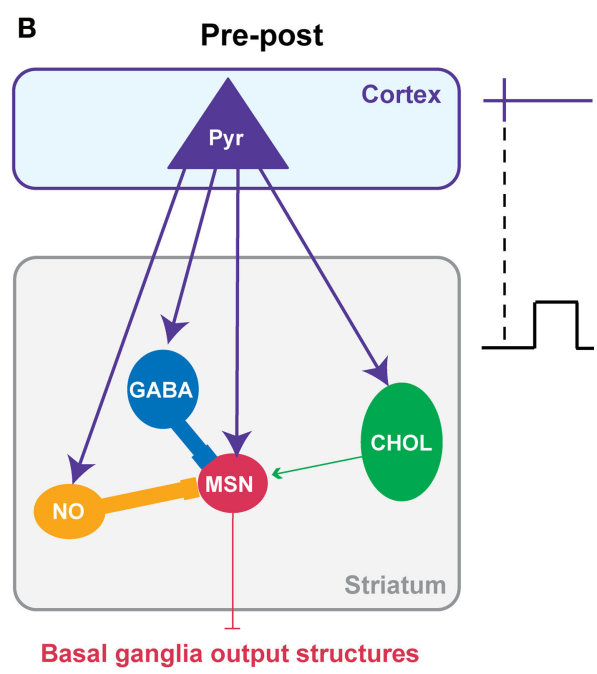

cholinergic interneurons. Consequently, it is expected that the synaptic weight of the striatal output would be increased. (B) Conversely, pre-post pairings lead to a potentiation of the GABAergic inhibition exerts by interneurons on MSNs while synaptic weight of MSNs and cholinergic interneurons decreases. Consequently, it is expected that the striatal output would be decreased. In conclusion, it appears that striatal STDP occurring at the different striatal neuronal population would act synergistically to increase or decrease the striatal output. 
characterized as following: GABAergic and nNOS interneurons have a strong inhibitory weight on MSNs and we will consider here the excitatory effect of cholinergic interneurons (Figure 2).

For a post-pre corticostriatal paired activity, the effect of the potentiation of the MSN synaptic efficacy would then be reinforced by the decreased inhibitory weight of GABAergic and nNOS interneurons. In addition, the excitatory effect of cholinergic interneurons would be increased by the induction of t-LTP at their level. Therefore, all these synaptic efficacy changes would work in synergy to increase the striatal output (Figure 5A). Conversely, after pre-post pairings, the corticostriatal transmission is depressed for MSNs, and this would be accentuated by the increase of the inhibition from GABAergic and nNOS interneurons and the decrease of excitatory effect of cholinergic interneurons (Figure 5B). In conclusion, the STDP of striatal interneurons and MSNs would act together to either increase or depress the striatal output.

Of course this scheme is quite simple and do not consider all the fine regulation and the specificity of STDP within the striatum. Indeed, it only considers the excitatory effect of cholinergic interneurons onto MSNs via M1 receptors, although an inhibitory effect mediated by M4 receptors has been also reported (Acquas and DiChiara, 2002). In addition, even though post-pre sequences induced a majority of t-LTP at cholinergic interneurons, they also induced t-LTD; the occurrence of t-LTP or t-LTD was dependent on the excitability of the cells (Fino et al., 2008). We should also consider that nNOS interneurons displayed a specific STDP timedependence since for pre-post sequences they develop t-LTD for short $\Delta \mathrm{t}$ and $\mathrm{t}$-LTP for longer $\Delta \mathrm{t}$ (Fino et al., 2009b). Finally, we will need to consider the strong effect of local modulation within the striatum like the dopaminergic afferents from the SNc for example (Nicola et al., 2000; Costa, 2007). Nevertheless, this scheme helps to understand how all these STDP interact synergistically. These results show that it is very important to consider the striatal neuronal heterogeneity to understand properly the integration of the cortical information by the striatum and its transmission toward the basal ganglia output structures.

\section{SUBTHRESHOLD EVENTS ACT AS HEBBIAN SIGNAL FOR CORTICOSTRIATAL LONG-TERM PLASTICITY: THE SUBTHRESHOLD-DEPOLARIZATION DEPENDENT PLASTICITY}

In the current conception of activity-dependent plasticity, as highlighted by STDP, the action potential constitutes the physiologically pertinent coding event determinant for the induction of long-term synaptic plasticity. However, neuronal activity does not lead systematically to an action potential but also, in many cases, to subthreshold events. Accordingly, experimental data suggested that the back-propagating action potential would not be the only post-synaptic depolarizing event necessary for the induction of long-term synaptic plasticity. In the hippocampus, a low-frequency stimulation at $1 \mathrm{~Hz}$ induced exclusively LTD whatever the amplitude of post-synaptic depolarization (subthreshold EPSP versus action potential) (Staubli and Ji, 1996); the amplitude of post-synaptic depolarization only influencing the LTD magnitude. Changes of holding membrane potential for relatively long duration (1 min; Artola et al., 1990 and 250 ms; Sjöström et al., 2004) paired with theta burst or action potential, respectively, induced either LTP (Artola et al., 1990) or LTD (Sjöström et al., 2004).
Due to MSN electrophysiological properties (Calabresi et al., 1987; Nisenbaum and Wilson, 1995), cortical inputs do not systematically trigger an action potential but a wide range of post-synaptic depolarizations, which mostly remain subthreshold (Wilson, 1995; Stern et al., 1997, 1998; Mahon et al., 2006) (Figure 6A). Therefore, considering the role of striatum in sensorimotor and cognitive learning, the implication of subthreshold signals in long-term coding at MSN corticostriatal synapses would be determinant. Moreover, a subthreshold depolarization back-propagates very efficiently in the dendritic tree of the MSNs and modulates the activity of voltage-sensitive calcium channels (Carter et al., 2007). Subthreshold depolarization in MSNs, paired with a quasi-coincident cortical activity, are able to induce longterm synaptic plasticity, named "subthreshold-depolarization dependent plasticity" (SDDP with "sd" coding for subthreshold depolarization) (Fino et al., 2009a) (Figures 6B,C). The induction protocol was similar than the one for corticostriatal STDP: same duration of depolarization (30 ms), same stimulation frequency but with the post-synaptic depolarization remaining subthreshold. Pre-post pairings (subthreshold depolarization evoked just after a cortical stimulation) induce mainly sd-LTD and post-pre pairings (subthreshold depolarization evoked just before a cortical stimulation) induce either sd-LTP or sd-LTD. The occurrence of sd-LTP and sd-LTD was dependent on the level of MSN excitability. Comparison of corticostriatal SDDP and STDP indicates that a post-synaptic subthreshold depolarization is sufficient to induce bidirectional long-term plasticity while a post-synaptic action potential appears to be determinant in the strict orientation of the plasticity and the precision of the time window (Fino et al., 2009a) (Figure 6C). The same receptors are involved in the induction of corticostriatal STDP and SDDP since sd-LTD is dependent of CB1-receptor activation and sd-LTP requires the activation of NMDA receptors. Such similar pharmacology indicates that subthreshold events are very efficiently transmitted throughout the dendritic tree in MSNs since, in coincidence with a presynaptic activation, they are able to activate NMDA receptors or to induce a release of endocannabinoids similarly to a back-propagating action potential.

SDDP demonstrates that MSNs have the capability to fully take into account post-synaptic subthreshold signals paired with cortical activity and, depending on the timing between these activities and on neuronal excitability, to generate robust sd-LTD or sdLTP. SDDP could have multiple consequences for corticostriatal transmission. Thus, change in the corticostriatal transmission efficacy induced by SDDP is expected to shift the threshold of MSN coincidence detection and firing. Indeed, LTP induced by theta burst in the hippocampus has been shown to facilitate the coincidence detection (Xu et al., 2006). In MSNs, SDDP should therefore induce a temporal shift of the spike timing and consequently modify the occurrence and magnitude of a subsequent STDP. Such impact of SDDP on STDP is reinforced by the fact that STDP is highly temporally restricted and the temporal position of the action potential has a determinant weight on the induced long-term plasticity orientation and magnitude. In conclusion, SDDP extends considerably the capabilities of neuronal long-term coding, beyond the action potential, making the neuron a genuine analogue element. 


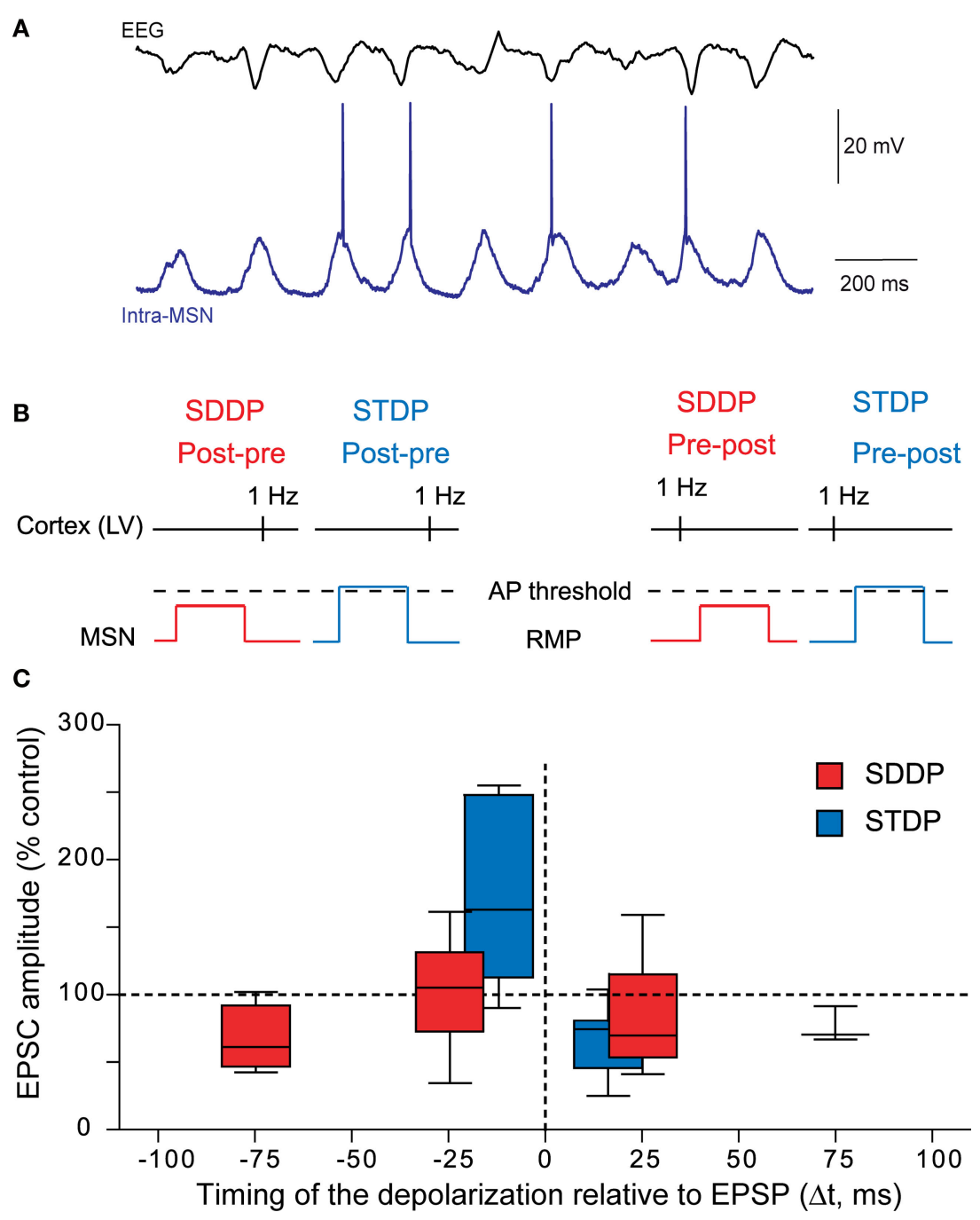

FIGURE 6 | Beyond the action potential, the subthreshold-depolarization dependent plasticity. (A) In vivo simultaneous recordings of the EEG of the cortical activity and the intracellular recording of one MSN. Synchronous cortical activity triggers action potentials in the MSN but also leads to subthreshold events (Adapted from Mahon et al., 2001). (B) Schematic representations of post-pre and pre-post SDDP and STDP protocols (the two protocols differ by the presence or not of a post-synaptic action potential). (C) Comparison of the occurrence, orientation, magnitude and temporal extent of SDDP and STDP. Long-term synaptic efficacy changes evoked by SDDP and STDP protocols illustrated with Box and Whiskers plots. When compared to SDDP, STDP changes were strictly orientated. SDDP changes were inducible in wider time windows than STDP ( \pm 110 vs. \pm 30 ms). (Adapted from Fino et al., 2009a).

\section{CONCLUSION}

The characterization of the different forms of non-synaptic and synaptic plasticity at the corticostriatal pathway constitutes a step toward the goal of understanding the cortico-basal ganglia information processing and the cellular basis of procedural learning. Nevertheless, to fully understand the modality of striatal plasticity and therefore the strength of the coincidence detection operated by MSNs, it will be determinant to consider the heterogeneity of the striatal compartments, the effects of neuromodulators (dopamine, serotonine, acetylcholine) and fully integrate the diversity of the neuronal sub-populations. In addition, although the corticostriatal pathway constitutes the main input pathway of the basal ganglia responsible for the selection of behaviorally pertinent information, it will be necessary to explore the synaptic plasticity occurring at the basal ganglia nuclei located downstream of the striatum to have the full picture of the successive plasticities all along cortico-basal ganglia information processing. Namely, STDP should be also investigated at relay (GPe) or output ( $\mathrm{SNr}$ and GPi) nuclei (Figure 1). Lastly, it remains to investigate in vivo the genuine impact of the corticostriatal STDP on the selection of cortical and thalamic activity. In vivo investigation of the effect of corticostriatal STDP during natural behaviors will indeed constitute a key step in understanding the cellular and synaptic mechanisms underlying procedural learning and memory in the basal ganglia.

\section{ACKNOWLEDGMENTS}

This work was supported by the INSERM and Collège de France. 


\section{REFERENCES}

Acquas, E., and DiChiara, G. (2002). Dopamine-Acetylcholine interactions. Handb. Exp. Pharmacol. 154, 85-115.

Aosaki, T., Graybiel, A. M., and Kimura, M. (1994). Effect of the nigrostriatal dopamine system on acquired neural responses in the striatum of behaving monkeys. Science 265, 412-415.

Apicella, P. (2007). Leading tonically active neurons of the striatum from reward detection to context recognition. Trends Neurosci. 30, 299-306.

Artola, A., Brocher, S., and Singer, W. (1990). Different voltage-dependent thresholds for inducing long-term depression and long-term potentiation in slices of rat visual cortex. Nature 347, 69-72.

Bennett, B. D., and Bolam, J. P. (1994). Synaptic input and output of parvalbumin-immunoreactive neurons in the neostriatum of the rat. Neuroscience 62, 707-719.

Bi, G. Q., and Rubin, J. (2005). Timing in synaptic plasticity: from detection to integration. Trends Neurosci. 28, 222-228.

Bliss, T. V., and Collingridge, G. L. (1993). A synaptic model of memory: longterm potentiation in the hippocampus. Nature 361, 31-39.

Bonsi, P., De Persis, C., Calabresi, P., Bernardi, G., and Pisani, A. (2004). Coordinate high-frequency pattern of stimulation and calcium levels control the induction of LTP in striatal cholinergic interneurons. Learn. Mem. 11, 755-760.

Calabresi, P., Gubellini, P., Centonze, D., Sancesario, G., Morello, M., Giorgi, M., Pisani, A., and Bernardi, G. (1999). A critical role of the nitric oxide/cGMP pathway in corticostriatal long-term depression. J. Neurosci. 19, 2489-2499.

Calabresi, P., Maj, R., Pisani, A., Mercuri, N. B., and Bernardi, G. (1992a). Long-term synaptic depression in the striatum: physiological and pharmacological characterization. J. Neurosci. $12,4224-4233$

Calabresi, P., Pisani, A., Mercuri, N. B., and Bernardi, G. (1992b). Longterm potentiation in the striatum is unmasked by removing the voltagedependent magnesium block of NMDA receptor channels. Eur. J. Neurosci. 4, 929-935.

Calabresi, P., Mercuri, N. B., and Di Filippo, M. (2009). Synaptic plasticity, dopamine and Parkinson's disease: one step ahead. Brain 132, 285-287.

Calabresi, P., Misgeld, U., and Dodt, H. U. (1987). Intrinsic membrane properties of neostriatal neurons can account for their low level of spontaneous activity. Neuroscience 20, 293-303.
Calabresi, P., Pisani, A., Mercuri, N. B., and Bernardi, G. (1996). The corticostriatal projection: from synaptic plasticity to dysfunctions of the basal ganglia. Trends Neurosci. 19, 19-24.

Caporale, N., and Dan, Y. (2008). Spike timing-dependent plasticity: a Hebbian learning rule. Annu. Rev. Neurosci. 31, 25-46.

Carter, A. G., Soler-Llavina, G. J., and Sabatini, B. L. (2007). Timing and location of synaptic inputs determine modes of subthreshold integration in striatal medium spiny neurons. $J$. Neurosci. 27, 8967-8977.

Centonze, D., Gubellini, P., Pisani, A. Bernardi, G., and Calabresi, P. (2003). Dopamine, acetylcholine and nitric oxide systems interact to induce corticostriatal synaptic plasticity. Rev. Neurosci. 14, 207-216.

Clarke, P. B., and Pert, A. (1985). Autoradiographic evidence for nicotine receptors on nigrostriatal and mesolimbic dopaminergic neurons. Brain Res. 348, 355-358.

Costa, R. M. (2007). Plastic corticostriatal circuits for action learning: what's dopamine got to do with it? Ann. N. Y. Acad. Sci. 1104, 172-191.

Dan, Y., and Poo, M. M. (2004). Spike timing-dependent plasticity of neural circuits. Neuron 44, 23-30.

Dan, Y., and Poo, M. M. (2006). Spike timing-dependent plasticity: from synapse to perception. Physiol. Rev. 86, 1033-1048.

Deniau, J. M., and Thierry, A. M. (1997). Anatomical segregation of information processing in the rat substantia nigra pars reticulata. Adv. Neurol. 74, 83-96.

Di Filippo, M., Picconi, B., Tantucci, M., Ghiglieri, V., Bagetta, V., Sgobio, C. Tozzi, A., Parnetti, L., and Calabresi, P. (2009). Short-term and longterm plasticity at corticostriatal synapses: implications for learning and memory. Behav. Brain Res. 199, 108-118.

Ding, J., Peterson, J. D., and Surmeier, D. J. (2008). Corticostriatal and thalamostriatal synapses have distinctive properties. J. Neurosci. 28, 6483-6492.

Egger, V., Feldmeyer, D., and Sakmann, B. (1999). Coincidence detection and changes of synaptic efficacy in spiny stellate neurons in rat barrel cortex. Nat. Neurosci. 2, 1098-1105.

Exley, R., and Cragg, S. J. (2008). Presynaptic nicotinic receptors: a dynamic and diverse cholinergic filter of striatal dopamine neurotransmission. Br. J. Pharmacol. 153(Suppl. 1), S283-S297.

Figueredo-Cardenas, G., Medina, L., and Reiner, A. (1996). Calretinin is largely localized to a unique population of striatal interneurons in rats. Brain Res. 709, 145-150.

Fino, E., Deniau, J. M., and Venance, L. (2008). Cell-specific spike-timingdependent plasticity in GABAergic and cholinergic interneurons in corticostriatal rat brain slices. J. Physiol. (Lond.) 586, 265-282.

Fino, E., Deniau, J. M., and Venance, L. (2009a). Brief subthreshold events can act as Hebbian signals for longterm plasticity. PLoS ONE 4, e6557. doi:10.1371/journal.pone.0006557.

Fino, E., Paille, V., Deniau, J. M., and Venance, L. (2009b). Asymmetric spike-timing dependent plasticity of striatal nitric oxide-synthase interneurons. Neuroscience 160, 744-754.

Fino, E., Glowinski, J., and Venance, L. (2005).Bidirectional activity-dependent plasticity at corticostriatal synapses. J. Neurosci. 25, 11279-11287.

Fino, E., Paille, V., Cui, Y., MoreraHerreras, T., Deniau, J., and Venance, L. (2010). Distinct coincidence detectors govern the corticostriatal sipke timing dependent plasticity. J. Physiol. (Lond.). (in press).

Gerfen, C. R. (1992). The neostriatal mosaic: multiple levels of compartmental organization in the basa ganglia. Annu. Rev. Neurosci. 15 285-320.

Graybiel,A.M. (1990). Neurotransmitters and neuromodulators in the basal ganglia. Trends Neurosci. 13, 244-254.

Graybiel, A. M. (2005). The basal ganglia: learning new tricks and loving it. Curr. Opin. Neurobiol. 15, 638-644.

Graybiel, A. M., Aosaki, T., Flaherty, A. W., and Kimura, M. (1994). The basal ganglia and adaptive motor control. Science 265, 1826-1831.

Groenewegen, H. J., and Berendse, H. W (1994). The specificity of the 'nonspecific' midline and intralaminar thalamic nuclei. Trends Neurosci. 17, 52-57.

Groenewegen, H. J., Berendse, H. W., Wolters, J. G., and Lohman, A. H. (1990). The anatomical relationship of the prefrontal cortex with the striatopallidal system, the thalamus and the amygdala: evidence for a parallel organization. Prog. Brain Res. 85, 95-116; discussion 116-118.

Kawaguchi, Y. (1993). Physiological, morphological, and histochemical characterization of three classes of interneurons in rat neostriatum. $J$. Neurosci. 13, 4908-4923.

Kawaguchi, Y., Wilson, C. J., and Emson, P. C. (1990). Projection subtypes of rat neostriatal matrix cells revealed by intracellular injection of biocytin. J. Neurosci. 10, 3421-3438.

Kimura, M., Yamada, H., and Matsumoto, N. (2003). Tonically active neurons in the striatum encode motivational contexts of action. Brain Dev. 25(Suppl. 1), S20-S23.

Kita, H. (1996). Glutamatergic and GABAergic postsynaptic responses of striatal spiny neurons to intrastriatal and cortical stimulation recorded in slice preparations. Neuroscience 70, 925-940.

Kita, H., Kosaka, T., and Heizmann, C.W. (1990). Parvalbumin-immunoreactive neurons in the rat neostriatum: a light and electron microscopic study. Brain Res. 536, 1-15.

Koos, T., and Tepper,J.M.(1999).Inhibitory control of neostriatal projection neurons by GABAergic interneurons. Nat. Neurosci. 2, 467-472.

Kreitzer, A. C., and Malenka, R. C. (2008). Striatal plasticity and basal ganglia circuit function. Neuron 60 , 543-554.

Kubota, Y., and Kawaguchi, Y. (2000). Dependence of GABAergic synaptic areas on the interneuron type and target size. J. Neurosci. 20, 375-386.

Levesque, M., and Parent, A. (2005). The striatofugal fiber system in primates: a reevaluation of its organization based on single-axon tracing studies. Proc. Natl. Acad. Sci. U.S.A. 102, 11888-11893.

Lin, J.Y., Chung, K. K., de Castro, D., Funk, G. D., and Lipski, J. (2004). Effects of muscarinic acetylcholine receptor activation on membrane currents and intracellular messengers in medium spiny neurones of the rat striatum. Eur. J. Neurosci. 20, 1219-1230.

Lynch, M. A. (2004). Long-term potentiation and memory. Physiol. Rev. 84, 87-136.

Mahon, S., Deniau, J. M., and Charpier, S. (2001). Relationship between EEG potentials and intracellular activity of striatal and cortico-striatal neurons: an in vivo study under different anesthetics. Cereb. Cortex 11, 360-373.

Mahon, S., Deniau, J. M., and Charpier, S. (2004). Corticostriatal plasticity: life after the depression. Trends Neurosci. 27, 460-467.

Mahon, S., Vautrelle, N., Pezard, L., Slaght, S. J., Deniau, J. M., Chouvet, G., and Charpier, S. (2006). Distinct patterns of striatal medium spiny neuron activity during the natural sleep-wake cycle. J. Neurosci. 26, 12587-12595.

Malenka, R. C., and Bear, M. F. (2004). LTP and LTD: an embarrassment of riches. Neuron 44, 5-21.

Mallet, N., Le Moine, C., Charpier, S., and Gonon, F. (2005). Feedforward inhibition of projection neurons by fast-spiking GABA interneurons in the rat striatum in vivo. J. Neurosci. $25,3857-3869$ 
Markram, H., Lubke, J., Frotscher, M. and Sakmann, B. (1997). Regulation of synaptic efficacy by coincidence of postsynaptic APs and EPSPs. Science 275, 213-215.

Martin, S. J., and Morris, R. G. (2002). New life in an old idea: the synaptic plasticity and memory hypothesis revisited. Hippocampus 12, 609-636.

Matamales, M., Bertran-Gonzalez, J., Salomon, L., Degos, B., Deniau, J. M., Valjent, E., Herve, D., and Girault, J.A. (2009). Striatal medium-sized spiny neurons: identification by nuclear staining and study of neuronal subpopulations in BAC transgenic mice. PLoS ONE4, e4770. doi:10.1371/journal.pone.0004770.

Morris, G., Arkadir, D., Nevet, A., Vaadia, E., and Bergman, H. (2004). Coincident but distinct messages of midbrain dopamine and striatal tonically active neurons. Neuron 43, 133-143.

Nicola, S. M., Surmeier, J., and Malenka, R. C. (2000). Dopaminergic modulation of neuronal excitability in the striatum and nucleus accumbens. Annu. Rev. Neurosci. 23, 185-215.

Nisenbaum, E. S., and Wilson, C. J. (1995). Potassium currents responsible for inward and outward rectification in rat neostriatal spiny projection neurons. J. Neurosci. 15, 4449-4463.

Nisenbaum, E. S., Xu, Z. C., and Wilson, C. J. (1994). Contribution of a slowly inactivating potassium current to the transition to firing of neostriatal spiny projection neurons. J. Neurophysiol. 71, 1174-1189.

Packard, M. G., and Knowlton, B. J. (2002). Learning and memory functions of the Basal Ganglia. Annu. Rev. Neurosci. 25, 563-593.

Pakhotin, P., and Bracci, E. (2007). Cholinergic interneurons control the excitatory input to the striatum. $J$. Neurosci. 27, 391-400.

Partridge, J. G., Apparsundaram, S., Gerhardt, G. A., Ronesi, J., and Lovinger, D. M. (2002). Nicotinic acetylcholine receptors interact with dopamine in induction of striatal long-term depression. J. Neurosci. 22, 2541-2549.

Pawlak, V., and Kerr, J. N. (2008). Dopamine receptor activation is required for corticostriatal spike-timing-dependent plasticity. J. Neurosci. 28, 2435-2446.

Perez-Rosello, T., Figueroa, A., Salgado, H., Vilchis, C., Tecuapetla, F., Guzman, J. N., Galarraga, E., and Bargas, J. (2005). Cholinergic control of firing pattern and neurotransmission in rat neostriatal projection neurons: role of
CaV2.1 and CaV2.2 Ca2+ channels. I. Neurophysiol. 93, 2507-2519.

Plenz, D., and Kitai, S. T. (1998). Up and down states in striatal medium spiny neurons simultaneously recorded with spontaneous activity in fast-spiking interneurons studied in cortex-striatum-substantia nigra organotypic cultures. J. Neurosci. 18, 266-283.

Ramanathan, S., Hanley, J. J., Deniau, J. M., and Bolam, J. P. (2002). Synaptic convergence of motor and somatosensory cortical afferents onto GABAergic interneurons in the rat striatum. $J$. Neurosci. 22, 8158-8169.

Redgrave, P., and Gurney, K. (2006). The short-latency dopamine signal: a role in discovering novel actions? Nat. Rev. Neurosci. 7, 967-975.

Reynolds, J. N., and Wickens, J. R. (2004). The corticostriatal input to giant aspiny interneurons in the rat: a candidate pathway for synchronising the response to reward-related cues. Brain Res. 1011, 115-128.

Sardo,P.,Ferraro, G., Di Giovanni, G., Galati, S., and La Grutta, V. (2002). Inhibition of nitric oxide synthase influences the activity of striatal neurons in the rat. Neurosci. Lett. 325, 179-182.

Schultz, W. (2007). Behavioral dopamine signals. Trends Neurosci. 30, 203-210.

Sergeeva, O. A., Doreulee, N., Chepkova, A. N., Kazmierczak, T., and Haas, H. L. (2007). Long-term depression of cortico-striatal synaptic transmission by DHPG depends on endocannabinoid release and nitric oxide synthesis. Eur. J. Neurosci. 26, 1889-1894.

Shen, W., Flajolet, M., Greengard, P., and Surmeier, D. J. (2008). Dichotomous dopaminergic control of striatal synaptic plasticity. Science 321, 848-851.

Sjöström, P. J., and Nelson, S. B. (2002). Spike timing, calcium signals and synaptic plasticity. Curr. Opin. Neurobiol. 12, 305-314.

Sjöström, P. J., Turrigiano, G. G., and Nelson, S.B.(2004). Endocannabinoiddependent neocortical layer-5 LTD in the absence of postsynaptic spiking. J. Neurophysiol. 92, 3338-3343.

Smeal, R. M., Gaspar, R. C., Keefe, K. A., and Wilcox, K. S. (2007). A rat brain slice preparation for characterizing both thalamostriatal and corticostriatal afferents. J. Neurosci. Methods 159, 224-235.

Smith, Y., Raju, D. V., Pare, J. F., and Sidibe, M. (2004). The thalamostriatal system: a highly specific network of the basal ganglia circuitry. Trends Neurosci. 27, 520-527.

Staubli,U.V., and Ji,Z.X. (1996). The induction of homo- vs. heterosynaptic LTD in area CA1 of hippocampal slices from adult rats. Brain Res. 714, 169-176.

Stern, E. A., Jaeger, D., and Wilson, C. J. (1998). Membrane potential synchrony of simultaneously recorded striatal spiny neurons in vivo. Nature 394, 475-478.

Stern, E. A., Kincaid, A. E., and Wilson, C. J. (1997). Spontaneous subthreshold membrane potential fluctuations and action potential variability of rat corticostriatal and striatal neurons in vivo. J. Neurophysiol. 77, 1697-1715.

Surmeier, D. J., Ding, J., Day, M., Wang, Z., and Shen, W. (2007). D1 and D2 dopamine-receptor modulation of striatal glutamatergic signaling in striatal medium spiny neurons. Trends Neurosci. 30, 228-235.

Suzuki, T., Miura, M., Nishimura, K., and Aosaki, T. (2001). Dopaminedependent synaptic plasticity in the striatal cholinergic interneurons. $J$. Neurosci. 21, 6492-6501.

Tepper, J. M., and Bolam, J. P. (2004) Functional diversity and specificity of neostriatal interneurons. Curr. Opin. Neurobiol. 14, 685-692.

Tepper, J. M., Koos, T., and Wilson, C. J. (2004). GABAergic microcircuits in the neostriatum. Trends Neurosci. 27, 662-669.

Thomas, T. M., Smith, Y., Levey, A. I., and Hersch, S. M. (2000). Cortical inputs to $\mathrm{m} 2$-immunoreactive striatal interneurons in rat and monkey. Synapse 37, 252-261.

Tzounopoulos, T., Kim, Y., Oertel, D., and Trussell, L. O. (2004). Cell-specific, spike timing-dependent plasticities in the dorsal cochlear nucleus. Nat. Neurosci. 7, 719-725.

Valjent, E., Bertran-Gonzalez, J., Herve, D., Fisone, G., and Girault, J.A. (2009) Looking BAC at striatal signaling: cellspecific analysis in new transgenic mice. Trends Neurosci. 32, 538-547.

Vandecasteele, M., Deniau, J. M., Glowinski, J., and Venance, L. (2007). Electrical synapses in basal ganglia. Rev. Neurosci. 18, 15-35.

Venance, L., Glowinski, J., and Giaume, C. (2004). Electrical and chemical transmission between striatal GABAergic output neurones in rat brain slices. $J$. Physiol. (Lond.) 559, 215-230.

Vuillet, J., Kerkerian, L., Kachidian, P. Bosler, O., and Nieoullon, A. (1989). Ultrastructural correlates of functional relationships between nigral dopaminergic or cortical afferent fibers and neuropeptide Y-containing neurons in the rat striatum. Neurosci. Lett. 100, 99-104.

Vuillet, J., Kerkerian-Le Goff, L., Kachidian, P., Dusticier, G., Bosler,
O., and Nieoullon, A. (1990). Striatal NPY-containing neurons receive GABAergic afferents and may also contain GABA: an electron microscopic study in the rat. Eur. J. Neurosci. 2, 672-681.

West, A. R., and Grace, A. A. (2004). The nitric oxide-guanylyl cyclase signaling pathway modulates membrane activity States and electrophysiological properties of striatal medium spiny neurons recorded in vivo. J. Neurosci. 24, 1924-1935.

Wilson, C. J. (1995). “The contribution of cortical neurons to the firing pattern of striatal spiny neurons," in Models of Information Processing in the Basal Ganglia, eds J. C. Houk, J. L. Davies, and D. G. Beiser (Cambridge, MIT Press), 29-50.

Wu, Y., Richard, S., and Parent, A. (2000). The organization of the striatal output system: a single-cell juxtacellular labeling study in the rat. Neurosci. Res. 38, 49-62.

Xu, N.L., Ye, C. Q., Poo, M. M., and Zhang, X. H. (2006). Coincidence detection of synaptic inputs is facilitated at the distal dendrites after long-term potentiation induction. J. Neurosci. 26, 3002-3009.

Yin, H. H., and Knowlton, B. J. (2006). The role of the basal ganglia in habit formation. Nat. Rev. Neurosci. 7, 464-476.

Yin, H. H., Mulcare, S. P., Hilario, M. R., Clouse, E., Holloway, T., Davis, M. I., Hansson, A. C., Lovinger, D. M., and Costa, R. M. (2009). Dynamic reorganization of striatal circuits during the acquisition and consolidation of a skill. Nat. Neurosci. 12, 333-341.

Conflict of Interest Statement: The authors declare that the research was conducted in the absence of any commercial or financial relationship that could be construed as a potential conflict of interest.

Received: 08 February 2010; paper pending published: 14 March 2010; accepted: 17 May 2010; published online: 10 June 2010.

Citation: Fino E and Venance L (2010) Spike-timing dependent plasticity in the striatum. Front. Syn. Neurosci. 2:6. doi: 10.3389/fnsyn.2010.00006

Copyright (C) 2010 Fino and Venance. This is an open-access article subject to an exclusive license agreement between the authors and the Frontiers Research Foundation, which permits unrestricted use, distribution, and reproduction in any medium, provided the original authors and source are credited. 